\title{
Quantifying the errors due to overfilling for Newtonian fluids in rotational rheometry
}

\author{
Ruth Cardinaels $^{1}$ (D) . Naveen Krishna Reddy ${ }^{2,3} \cdot$ Christian Clasen $^{4}$
}

Received: 13 January 2019 / Revised: 6 May 2019 / Accepted: 17 May 2019 / Published online: 6 July 2019

(C) The Author(s) 2019

\begin{abstract}
The errors on rheological measurements due to overfilling of Newtonian fluids using parallel plate and cone-plate setups in rotational rheometry are quantified. Overfilled sample causes an additional drag force, thereby increasing the measured viscosity, especially when the sample wets the geometry rim. This can cause errors up to $30 \%$ in standard experimental setups such as parallel plates with a gap height of $1 \mathrm{~mm}$. This viscosity error increases proportionally with the ratio of gap height to radius of the geometry. By developing a scaling relation that captures the main effects of the geometrical parameters on the viscosity error due to overfilling, a master curve was constructed for the viscosity error as a function of the amount of overfilling. Our systematic analysis of the viscosity error due to overfilling can be utilized to correct for this error during rheological measurements in which overfilling is known but unavoidable or desired.
\end{abstract}

Keywords Overfilling $\cdot$ Edge effects $\cdot$ Rotational rheometry $\cdot$ Shear viscosity

\section{Introduction}

Rotational rheometers are ubiquitous in both academia and industry. In soft matter research, rotational rheometers are frequently used to characterize the flow behavior and moduli of complex materials. Using structure-rheology relations that are well-established for a wide range of material classes, rheology can also provide in situ and time-resolved microstructural information (Larson 1999). In industrial production units, rotational rheometers are commonly present for monitoring the quality of the produced materials. The most common measuring

Part of a Special Issue "Novel Trends in Rheology".

Ruth Cardinaels

R.M.Cardinaels@tue.nl

1 Polymer Technology, Department of Mechanical Engineering, Eindhoven University of Technology (TU Eindhoven), P.O. Box 513, 5600MB, Eindhoven, The Netherlands

2 Faculty of Industrial Engineering, Hasselt University, Martelarenlaan 42, 3500, Hasselt, Belgium

3 IMO, IMOMEC, Hasselt University, Wetenschapspark 1, 3590, Diepenbeek, Belgium

4 Soft Matter Rheology and Technology, Department of Chemical Engineering, KU Leuven, Celestijnenlaan 200F, Box 2424, 3001, Leuven, Belgium geometries in rotational rheometers are the parallel plate and cone-plate geometries with the bottom plate in many configurations being larger than the top plate (Macosko 1994). The analysis to extract rheological parameters from measurements with these geometries is based on the assumption of a cylindrical (for parallel plate setup) or spherical (for cone-plate setup) shape of the free surface of the sample (Macosko 1994). It is common knowledge in experimental rheology that it is essential to trim the sample edge carefully after sample loading and to maintain the spherical or cylindrical surface shape during actual measurements. Kalika et al. (1986) state that "the need to monitor the edge is part of the folklore of rheometry." In spite of the above statement, overfilling and underfilling scenarios are encountered quite frequently, sometimes due to experimenter's inexperience, and also as an unavoidable result of the design of the measurement setup or the sample properties, or as a means to resolve other measurement complications. Some examples in which overfilling is unavoidable are rotational rheometers with upper geometries that inhibit sample trimming due to the presence of a large solvent reservoir on top of the geometry, measurements of samples with large contact angles (Choi and Kim 2006; Srinivasan et al. 2013) or linear motion micro-rheometers (Clasen and McKinley 2004; Clasen et al. 2006). Furthermore, overfilling is frequently used to avoid evaporation in volatile samples for which an excess amount 
of fluid is loaded in the rheometer or for which the sample edge is covered with a non-volatile second fluid. Overfilling also provides an effective means to avoid edge fracture (Quinzani and Vallés 1986; Vrentas et al. 1991; Snijkers and Vlassopoulos 2011; Schweizer and Schmidheiny 2013).

When considering the fluid mechanics problem of flow in a rotational rheometer, inertia, gravity, surface tension and edge effects at the air-liquid interface are commonly neglected (Walters 1975; Macosko 1994). For the typical rotational speeds used in rotational rheometers $\left(<500 \mathrm{~s}^{-1}\right)$, the Reynolds number is low, justifying the assumption of Stokes flow. It is then the balance between surface tension and gravity that determines the shape of the free sample surface (Shipman et al. 1991). Shipman et al. (1991) show that the deviation from the theoretical cylindrical surface shape in parallel plate rheometers generally remains rather limited. Although this small shape deviation has implications for the measured normal stresses, torque measurements for medium to high viscous samples are normally insensitive to the shape of the free sample surface, provided that the correct volume of liquid is present. However, a recent study by Johnston and Ewoldt (2013) has shown that for low viscous samples, the effect of surface tension via contact line tension can be large, already for a slight rotational asymmetry of the sample edge. In the presence of surface-active components, also interfacial rheology of the free sample surface can contribute to the measured torque signal, even with a perfectly cylindrical or spherical edge shape (Sharma et al. 2011). Obviously, when large deviations from the theoretical surface shape occur, such as edge fracture, the obtained rheological parameters will no longer be correct, even in the absence of surfaceactive components (Walters 1975; Macosko 1994; Tanner and Keentok 1983).

The effects of overfilling on rheological measurements have been studied both experimentally and theoretically for cases in which the rotating plate or cone is inserted in a large container of fluid (Griffiths et al. 1969; Griffiths and Walters 1970; Paddon and Walters 1979; Quinzani and Vallés 1986; Vrentas et al. 1991; Khaliullin and Schieber 2009). Quinzani and Vallés (1986) performed both experiments and modeling on a modified cone-plate measuring device that was designed to avoid edge fracture, as shown in Fig. 1a. They determined the effect on the measured torque and normal force due to the presence of the stationary closed cup around the rotating cone. They found that the presence of sample in the gap between the rotating cone and the top lid of the stationary container contributes substantially to the measured torque, but does not affect the normal force. Vrentas et al. (1991) studied the case in which a sample reservoir is used in a parallel plate setup. Their setup is depicted in Fig. 1b. They demonstrate that the ratio of the diameter of the container to that of

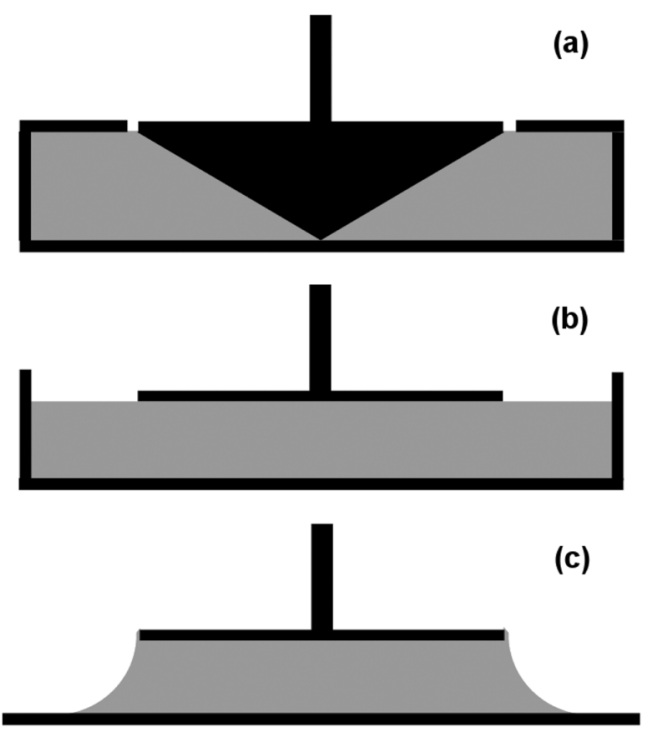

Fig. 1 Schematics of modified setups used for avoiding edge effects: a modified cone-plate fixture with closed side walls studied by Quinzani and Vallés (1986), b parallel plate setup with a sample reservoir studied by Vrentas et al. (1991), c typical overfilling scenario studied in the present work

the upper plate has a large effect on the measured torque, mainly when the container is not much larger than the plate. Similarly, Pieper and Schmid (2016) show that a guard ring around the sample affects both the velocity profile as well as the measured torque values. Other studies on overfilling consider a setup in which the fluid is filled in a large bath such that it also covers the top part of the measuring plate or cone, this is sometimes refereed to as the "sea of fluid configuration" (Paddon and Walters 1979; Griffiths et al. 1969; Griffiths and Walters 1970; Walters 1975).

To our knowledge, no systematic studies have been carried out on the situation in which a small amount of overfilling is present symmetrically around the measuring plate or cone in a regular rheometer as depicted in Fig. 1c. This is surprising as this represents the most common case of overfilling encountered. Giles and Hooper (1999) presented results showing that the effects of overfilling on the generated torque values in a rotational rheometer depend on the amount of overfilling, as well as on the ratio of gap spacing to plate diameter. Davies (2015) demonstrates that the viscosity measured with a cone-plate rheometer is less sensitive to overfilling as compared to underfilling. Nevertheless, this source of experimental error cannot just be taken for granted or ignored. We therefore present in this work a systematic study of the effects of overfilling for Newtonian fluids in rotational rheometry. Experiments are performed using both parallel plate and cone and plate geometries in a standard rotational rheometer with the bottom plate 
being much larger than the top geometry. The amount of overfilling is varied in a controlled way and correlated to the errors on the measured viscosity. Furthermore, the experimental results are compared with those of numerical simulations. The simulations provide insight into the origin of the measurement errors due to overfilling and allow to systematically study the effects of several geometrical parameters on the measurement errors due to overfilling. Finally, suggestions to minimize these errors are formulated from the perspectives of instrument design and execution of experiments.

\section{Materials and methods}

\section{Materials}

As Newtonian fluids, polydimethylsiloxanes (PDMS, Brookfield) with viscosities of $0.99,12.4$, and $101 \mathrm{~Pa}$ s at $25^{\circ} \mathrm{C}$ were used. For the fluid with the highest viscosity, a slight shear thinning sets in at shear rates above $10 \mathrm{~s}^{-1}$. In the present work, only shear rates in the range where the Brookfield oils have a constant viscosity are considered. The surface tension of polydimethylsiloxane at $25^{\circ} \mathrm{C}$ is approximately $20 \mathrm{mN} / \mathrm{m}$ (Wu 1982). Combined with the relatively high viscosity values, this ensures that the measured torque values will not be affected by surface tension effects (Johnston and Ewoldt 2013).

\section{Rheometry}

Rheological experiments were carried out using a stresscontrolled rheometer (Physica MCR 501 from Anton Paar) and a strain-controlled rheometer (ARES-LS from Rheometrics). Measurements were performed using a large bottom plate combined with a $25-$ or 50-mm-diameter top plate or cone (cone angles of $2^{\circ}(0.035 \mathrm{rad})$ and $5.7^{\circ}$ $(0.1 \mathrm{rad}))$. The parallel plate and cone-plate setups will be referred to in this work as the PP and CP setups. All geometries had smooth stainless steel surfaces as supplied by the manufacturer. The temperature was kept constant at $25{ }^{\circ} \mathrm{C}$ using a Peltier element integrated in the lower plate. An externally calibrated thermocouple (hypodermic needle, type T Copper-Constantan, Omega, Stamford, CT) was used to verify that the temperature in the sample was within $\pm 0.1^{\circ}$ of the set temperature. Steady-state flow experiments were carried out over a range of shear rates from 0.1 to $10 \mathrm{~s}^{-1}$ with a measurement time of $120 \mathrm{~s}$ at each shear rate. A minimum of two experiments with a new sample loading was performed for each overfilling case. The reported values are averages with the data precision of each point indicated by means of the standard deviation over the repeat measurements.

\section{Definition of viscosity error and amount of overfilling}

To quantify the effects of overfilling, the relative error in viscosity was determined. This error is defined as:

$\Delta \eta=\frac{\eta-\eta_{0 \%}}{\eta_{0 \%}}$

where $\eta$ is the measured viscosity with sample overfilling and $\eta_{0 \%}$ is the sample viscosity and thus the measured viscosity for an exact filling.

The amount of overfilling $\Delta \mathrm{OF}$ is defined as the ratio of the overfilled (=extra) volume to the volume required to exactly fill the geometry:

$\Delta \mathrm{OF}=\frac{V_{\mathrm{ex}}}{V_{\text {geometry }}}$

with:

$V_{\text {ex }}=V_{\text {sample }}-V_{\text {geometry }}$

wherein $V_{\text {sample }}$ is the sample volume and $V_{\text {geometry }}$ is the volume required to exactly fill the geometry. The volume required to exactly fill the geometry $V_{\text {geometry }}$ depends on the type of geometry, being equivalent to $H \pi R^{2}$ for the PP geometry, with $H$ the gap height and $R$ the plate radius. For the CP geometry, $V_{\text {geometry }}$ becomes:

$V_{\text {geometry,CP }}=\frac{2}{3} \pi R^{3} \tan (\theta)+\frac{1}{3} \pi \frac{H_{\text {trun }}^{3}}{\tan ^{2}(\theta)}$

with $R$ the cone radius, $\theta$ the cone angle and $H_{\text {trun }}$ the height of the truncated part of the cone tip. In this expression, the first term accounts for the volume around the cone whereas the second term accounts for the volume of the truncated part of the cone. The latter quantity is usually negligible due to the small value of $H_{\text {trun }}$.

\section{Loading procedures}

Specific loading procedures were used to obtain the required amounts of overfilling in a reproducible way. For all measurements, the sample under investigation was initially loaded onto the bottom plate. The upper geometry was then slowly moved down to the required gap. Samples with viscosity 12.4 Pa s were allowed to rest for $300 \mathrm{~s}$ before any measurement. In the case of no overfilling, it was ensured that the sample was precisely pinned to the edge of the geometry and did not touch the outer rim of the upper geometry during sample loading. For experiments using the PP setup, care was taken to avoid air bubble formation while bringing the upper plate to the required gap, as even low volume fractions of bubbles will affect the measurements (Joh et al. 2010; Tran-Duc et al. 2013). Air bubbles were avoided by placing a small amount of the sample to be measured in the center of the upper plate so as to induce a forced wetting 
from a single point. The presence of air bubbles during normal sample loading and their absence after using the forced wetting method were confirmed by visualization of the sample loading in an MCR301 rheometer equipped with a transparent bottom plate (Reddy 2019).

For measurements with the PP setup with varying gap height, the upper plate was initially brought to a gap of $1 \mathrm{~mm}$. The excess sample was then removed with a spatula. After this sample trimming, the exact sample volume $V_{\text {sample }}$ with a cylindrical surface was left underneath the plate. Subsequently, the plate was lowered to the smaller gap height $H$. A first set of experiments was performed at different gap heights for a fixed $V_{\text {sample, }}$, resulting in $\Delta \mathrm{OF}$ values between 0.05 and 1 . A second set of measurements with the PP setup was performed with a constant gap height of $0.5 \mathrm{~mm}$. In these experiments, the sample loading and trimming were performed at varying larger gaps, which were chosen as such that the loaded sample volume at the final height led to a $\triangle \mathrm{OF}$ between 0.05 and 1 . In the $\mathrm{CP}$ setup, the procedure was similar to that for the PP setup with constant gap height. In this case, the measurement gap was determined and fixed by the cone truncation. For the $\mathrm{CP}$ geometry, the correct sample edge shape in the absence of overfilling is spherical. To achieve this, the sample is trimmed at a slightly higher height before the upper cone geometry is brought to the desired measuring height $H_{\text {trun }}$ so that the sample at the edge of the $\mathrm{CP}$ geometry has the required spherical shape. The trimming height $H_{\text {trim }}$ in the cone-plate geometry can be obtained by determining the volume needed to provide a spherical sample edge, resulting in:

$H_{\text {trim }}=R(\theta-\sin \theta)+H_{\text {trun }}$

For larger degrees of overfilling, the sample will eventually not remain pinned to the lower edge of the geometry, but will also wet the outer rim and will pin to the upper edge of the rim. The occurrence of such a wetting was monitored, and in that case, a homogeneous wetting of the rim was assured. The occurrence of rim wetting was in particular taken into account for comparison with the numerical simulations.

\section{Numerical simulations}

Numerical simulations of the flow in parallel plate and cone-plate rotational rheometry setups were performed with the finite element package COMSOL Multiphysics. The simulation domain is shown in Fig. 2a. Due to the symmetry around the central axis, simulations in a $2 \mathrm{D}$ axisymmetric domain are sufficient to describe the full 3D flow. The boundary conditions consist of axial symmetry on the left boundary, a stationary wall with no slip at the bottom, a top wall with only a non-zero velocity component in the $\phi$ direction $\left(u_{\phi}(r)=\Omega r\right.$, with $\Omega$ the rotation speed and $r$ the radial distance from the center) and zero viscous stress on the right boundary that is in contact with air. The values of the angular velocity are chosen to provide shear rates that correspond to the experimental values. A zero pressure constraint is applied at the contact point between the bottom plate and the sample edge. The diameter of the top plate or
Fig. 2 a Schematic representation of simulation boundary conditions. b Four different configurations of the sample edge at the plate rim (not to scale). c Velocity distribution in the sheared sample with the two torque contributions indicated, where $M_{1}$ is due to the sample underneath the upper plate and $M_{2}$ is due to the sample in contact with the outer rim of the upper plate
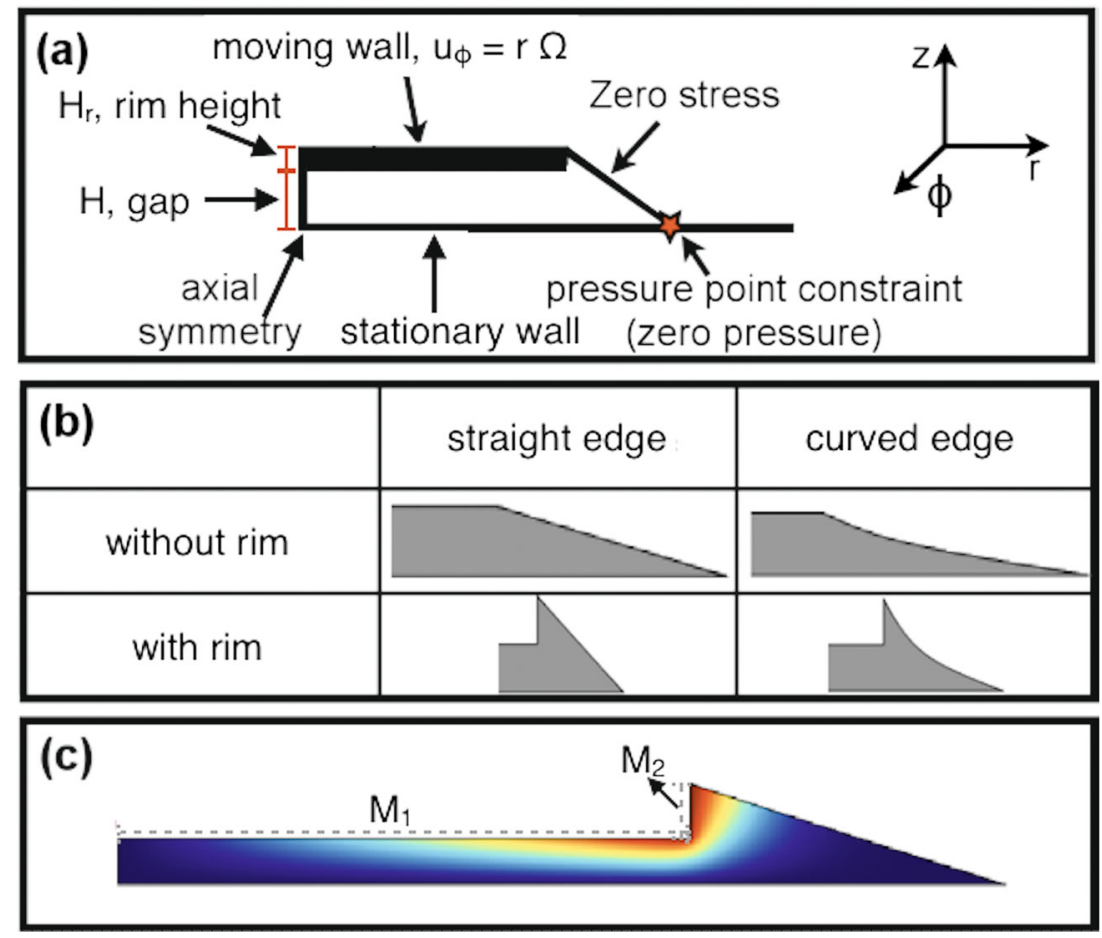
cone and the gap height are chosen equal to the experimental values. To study the effect of the shape of the sample edge on the outcome of rheological measurements, different edge shapes were imposed in the simulations, as illustrated in Fig. 2b. Two types of edge shapes are considered, each with and without including contact between the sample and the rim of the upper geometry. The first type is a straight boundary, starting from the edge of the upper plate and extending a certain distance along the lower plate, depending on the amount of overfilling. The second type of edge is a curved shape defined by a quadratic Bézier curve through the edge point of the upper plate (point 1), the sample contact point on the lower plate (point 3) and an intermediate point (point 2) for which the $r$ and $z$ coordinates obey the following relations:

$r_{2}=r_{1}+c\left(r_{3}-r_{1}\right)$

$z_{2}=d z_{1}$

whereby $c$ and $d$ determine the edge shape. The radial distance $r_{3}$ along the lower plate that is covered with sample is varied to obtain different amounts of overfilling. For Newtonian fluids and under stationary conditions, the following momentum and mass balance equations are solved in the fluid domain, with the boundary conditions as shown in Fig. 2a:

$\rho[(\mathbf{u} \cdot \nabla) \mathbf{u}]=\nabla \cdot\left[-p \mathbf{I}+\eta\left(\nabla \mathbf{u}+(\nabla \mathbf{u})^{T}\right)\right]+\mathbf{F}$

$\nabla \cdot \mathbf{u}=0$

in which $\eta$ is the viscosity, $p$ the pressure, $\mathbf{u}$ the velocity, I the identity tensor and $\mathbf{F}$ the external body force per unit volume. The viscosity is chosen equal to that of the polydimethylsiloxanes used in the experiments. The external body force consists of a gravitational force in the negative $z$-direction. The flow is assumed to be incompressible. The equations are discretized by means of a finite element method in the package COMSOL Multiphysics. A built-in predefined mesh type with triangular mesh elements calibrated for fluid dynamics is used, combined with 2 layers of quadrilateral boundary elements along the solid boundaries. It was verified that further refinement of the mesh did not significantly affect the obtained results. Piecewise linear functions are used to represent the pressure and the velocity is described by means of piecewise quadratic functions. The direct linear PARDISO solver is used, and in each simulation run, typically around 20,000 degrees of freedom are solved for in the PP setup and around 60,000 in the CP setup. Since the Reynolds number remains well below 1, numerical stabilization techniques were not necessary in the simulations.
From the simulated velocity fields, the torque $M$ on the upper plate can be obtained as:

$M=\int_{0}^{R} 2 \pi \eta_{0 \%} \dot{\gamma}_{\phi z}(r, H) r^{2} \mathrm{~d} r+\int_{H}^{H+H_{r}} 2 \pi \eta_{0 \%} \dot{\gamma}_{\phi r}(R, h) r^{2} \mathrm{~d} h$

in which $R$ is the radius of the upper plate, $H$ is the gap height, $H_{r}$ is the rim thickness of the upper plate, $\dot{\gamma}_{\phi z}(r, H)$ and $\dot{\gamma}_{\phi r}(R, h)$ are the shear rates at the lower boundary of the upper geometry and at the geometry rim respectively, with the subscripts $\phi z$ and $\phi r$ indicating the components of the rate of deformation tensor that correspond to the derivative of the $\phi$ direction velocity in either the $z$ or the $r$ direction (direction perpendicular to the respective wall), and $\eta_{0 \%}$ is the sample viscosity. The first term on the right-hand side of Eq. 10 is the contribution from the lower surface of the upper plate $\left(M_{1}\right.$ in Fig. $\left.2 \mathrm{c}\right)$ whereas the second term is the contribution of the side rim of the upper plate ( $M_{2}$ in Fig. 2c). The measured viscosity is then obtained from the standard relation between shear stress $\sigma$ and torque $M$ that is valid for a precisely filled rotational geometry (Macosko 1994):

$M=\frac{\sigma \pi R^{3}}{a}$

where $\sigma$ is the shear stress of the fluid at the rim and $a=2$ for PP geometries and $a=3 / 2$ for CP geometries (Macosko 1994). It was verified that this protocol leads to simulated viscosity values in the absence of overfilling that agree with the inserted sample viscosities. Moreover, since the main component of the rate of deformation tensor in the fluid inside the gap is the $\phi z$ component, whereas in the region against the edge of the upper geometry the $\phi r$ component dominates, the shear rates in Eq. 10 can be represented by the magnitude of the rate of deformation tensor.

\section{Experimental and numerical results}

The error on viscosity measurements in rotational rheometry due to overfilling of Newtonian liquids will be presented here. The experimental results for overfilling in various plate-plate and cone-plate setups will be presented in the "Experimental observations of viscosity error due to overfilling" section. Subsequently, the origins of the overfilling effects will be studied in more detail by means of numerical simulations in the "Origins of viscosity errors due to overfilling" section. Based on the insights gained in the "Origins of viscosity errors due to overfilling" section, all parameters playing a role in viscosity errors due to overfilling are captured in a single scaling relation in the "Master curves for viscosity error" section. 
Fig. 3 Images of the edge of the with a viscosity of $12.4 \mathrm{~Pa} \mathrm{~s}$ in a plate-plate setup with plate diameter $D=25 \mathrm{~mm}$ and gap height $H=0.5 \mathrm{~mm}$. a $\Delta \mathrm{OF}=$ 0.05 . b $\Delta \mathrm{OF}=0.10$. $\mathbf{c} \Delta \mathrm{OF}=$ $0.20 . \mathbf{d} \Delta \mathrm{OF}=0.40 . \mathbf{e} \Delta \mathrm{OF}=$ 0.70 overfilled sample for silicone oil

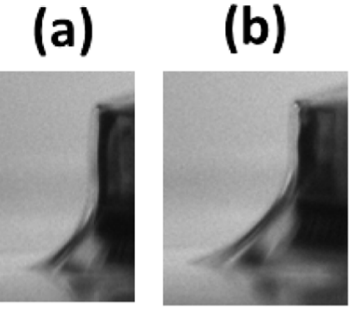

(c)
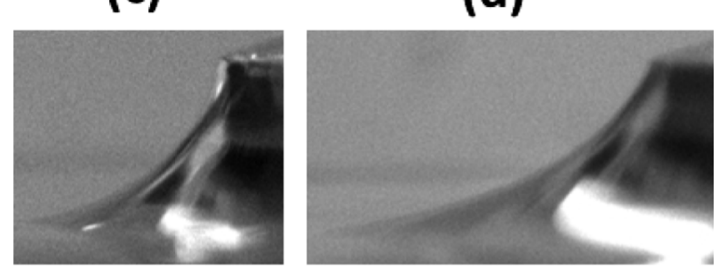

(e)

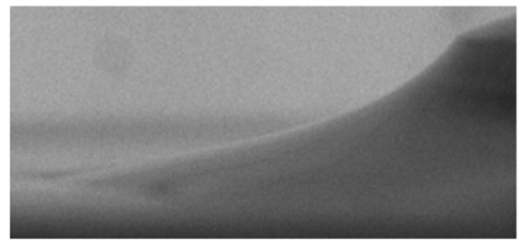

a constant final gap height, the viscosity error gradually increases and eventually plateaus. The data in Fig. 4 clearly show that overfilling can have a substantial effect on the measured viscosity values, with the plateau level of the viscosity error reaching up to $30 \%$. A comparison of the different datasets in Fig. 4 allows to conclude that the viscosity error substantially increases with both the gap height $H$ as well as the rim height $H_{\mathrm{r}}$. This can be rationalized by the fact that higher values of the gap height as well as the rim height result in a larger ratio of surface area in contact with overfilled sample as compared to the bottom surface area of the upper geometry. Since the later is the only surface on which the sample exhibits a torque

This has been the case in all experiments performed in this work, a pinning of the sample to only the lower edge of the upper geometry was not possible for any degree of overfilling above 0.2. For all samples, sufficient time was allowed for the sample edge to reach its equilibrium shape, which is determined by the pinning of the sample as well as its contact angle with the geometry and its surface tension. Due to the dominant contribution of viscous forces as compared with surface tension effects in the azimuthal direction, small asymmetries in the sample edge shape in this direction disappear due to the presence of the shear flow. It was verified that, once a symmetric surface shape was attained, the shear flow did no longer affect the shape of the sample edge, at least within the range of shear rates studied. This is expected to remain the case as long as inertia and elastic stresses are absent, since the viscous shear stress has no component in the direction of the edge curvature between top and bottom plate (Shipman et al. 1991).

Experimental viscosity errors obtained in plate-plate setups with a plate diameter of $25 \mathrm{~mm}$ are presented in Fig. 4. In all cases, the viscosity error is positive, indicating that the presence of extra, overfilled material around the geometry causes an additional torque exhibited on the upper geometry. When the measurements are performed at

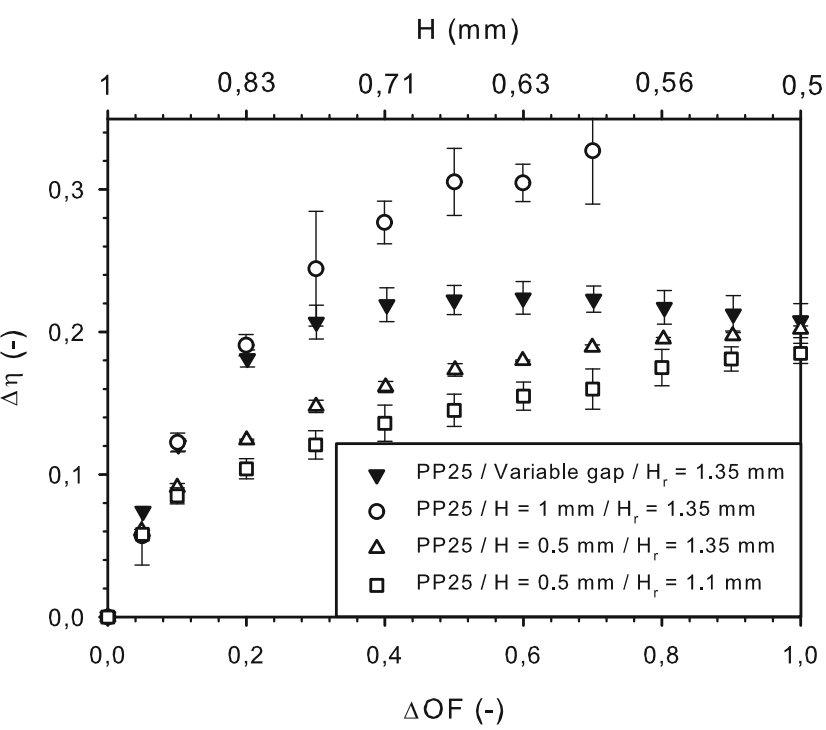

Fig. 4 Experimental data for the viscosity error due to overfilling of silicone oil with a viscosity of $12.4 \mathrm{~Pa} \mathrm{~s}$ in plate-plate setups with plate diameter $D=25 \mathrm{~mm}$ and various gap and rim heights $H$ and $H_{r}$. The error bars indicate the standard deviation over the repeat measurements 
in absence of overfilling whereas the former will generate extra torque contributions, increasing this ratio increases the viscosity error. When experiments are performed by gradually reducing the gap height, the viscosity error versus the amount of overfilling in Fig. 4 shows a small overshoot with increasing amount of overfilling. This can be attributed to the presence of two competing effects. While the amount of overfilling is increasing, the gap is simultaneously decreasing. For clarity, the gap height used in the variable gap experiments is shown on the top axis in Fig. 4. It can be seen that for a gap height of $0.5 \mathrm{~mm}$, exactly the same viscosity error is obtained whether this gap height is obtained via a direct reduction of the gap height from 1 to $0.5 \mathrm{~mm}$ or via the variable gap experiments. This is caused by the fact that in both cases the same shape of the overfilled sample was obtained.

Figure 5 shows the effect of plate diameter, whereby a larger plate diameter $(50 \mathrm{~mm}$ versus $25 \mathrm{~mm}$ ) results in a significantly smaller effect of overfilling on the viscosity measurements. Similar to the effects of gap height, this can be attributed to the relative contributions of surface area in contact with overfilled sample versus bottom surface of the upper geometry. Figure 5 also shows the effects of overfilling for cone-plate setups with a diameter of $25 \mathrm{~mm}$. To allow a direct comparison with the results for the plate-plate setups, the gap under the cone at the edge of the geometry is indicated as gap height in the figure. It can be seen that a cone-plate geometry with a similar diameter and gap height exhibits a smaller viscosity error as compared to the corresponding plate geometry. To estimate the significance of the viscosity error due to overfilling, it

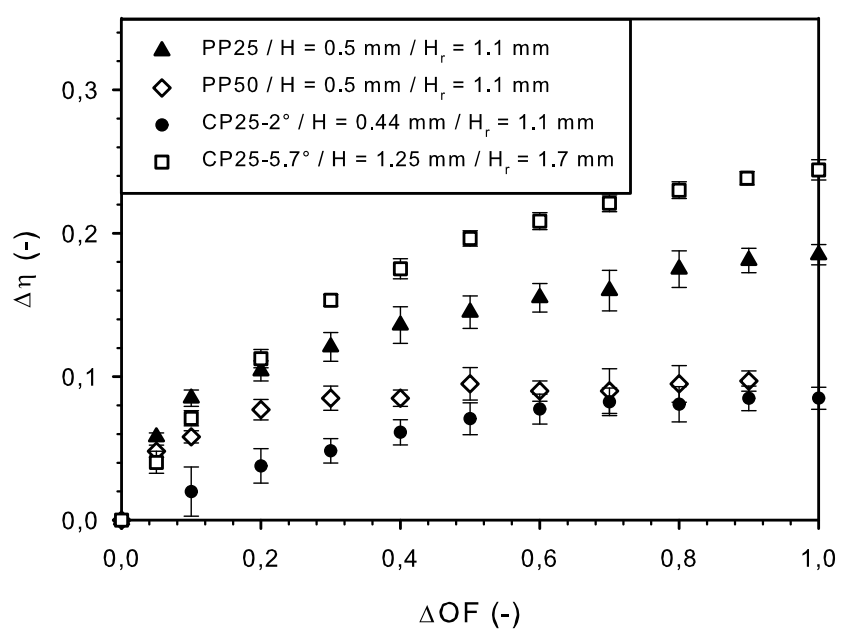

Fig. 5 Experimental data for the viscosity error due to overfilling of silicone oil with viscosity $12.4 \mathrm{~Pa} \mathrm{~s}$ in plate-plate setups with plate diameter $D=25 \mathrm{~mm}$ and $D=50 \mathrm{~mm}$ as well as cone-plate setups with cone diameter $D=25 \mathrm{~mm}$ and various cone angles $\theta$. The error bars indicate the standard deviation over the repeat measurements can be compared with the typical precision and accuracy of a viscosity measurement, which is within $\pm 1.5 \%$ for these Newtonian oils and when using a well-calibrated rheometer. The precision of the data is indicated by the error bars in Figs. 4 and 5 that indicate the standard deviation obtained from repeat measurements.

\section{Origins of viscosity errors due to overfilling}

Numerical simulations of the flow of Newtonian fluids in rotational rheometry have been performed to study the local effects of sample overfilling on the flow field. This in turn allows to shed light on the implications of the flow field alteration on the measured rheological parameters. The actual sample shape of the overfilled sample will depend on a balance between the surface forces and gravity (Bond number). However, our aim is to unravel the origin of the viscosity error due to overfilling and to provide insights in the dependence of this viscosity error on the relevant geometrical parameters. Therefore, we did not attempt to numerically solve the free-surface problem. Hence, the actual transient shaping process during which the sample shape evolves under the driving forces of gravity and surface forces resisted by the viscoelastic stresses inside the sample is beyond the scope of the present work. Rather, we fixed various steady state shapes of the overfilled volume and imposed a zero stress boundary condition on the sample edge to provide relevant insights. Figure 6 shows a comparison between the viscosity error obtained from numerical simulations and the experimentally determined one in a plate-plate setup with plate diameter $D=25 \mathrm{~mm}$. The simulation data correspond to the different overfill scenarios defined in Fig. 2b. When the excess amount of sample wetting the upper plate rim is not taken into account, the predicted viscosity error in Fig. 6 is much smaller than in reality (5\% versus approximately $20 \%$ error), thereby indicating that the extra fluid wetting the upper geometry rim has a large contribution to the viscosity error. The upper four simulated curves all include the fluid against the plate rim, but with a different shape corresponding to different values of $c$ and $d$ in Eqs. 6 and 7. It can clearly be seen that changing the shape of the sample edge results in moderate effects on the viscosity error as compared to the difference between the situations with and without rim. The simulation results in Fig. $6 \mathrm{a}$ and the selection of edge shapes at $\Delta \mathrm{OF}=0.50$ in Fig. $6 \mathrm{~b}$ clearly demonstrate that the straight edge scenario, that carries more fluid close to the rim for the same $\Delta \mathrm{OF}$ as compared with the scenario with the curved edge, leads to a slightly higher viscosity error in Fig. 6a. This can be attributed to the fact that the extra fluid on the bottom plate that extends far from the actual sample does not contribute significantly to the measured torque. From Fig. 6a, it can be concluded that when the sample 
(a)

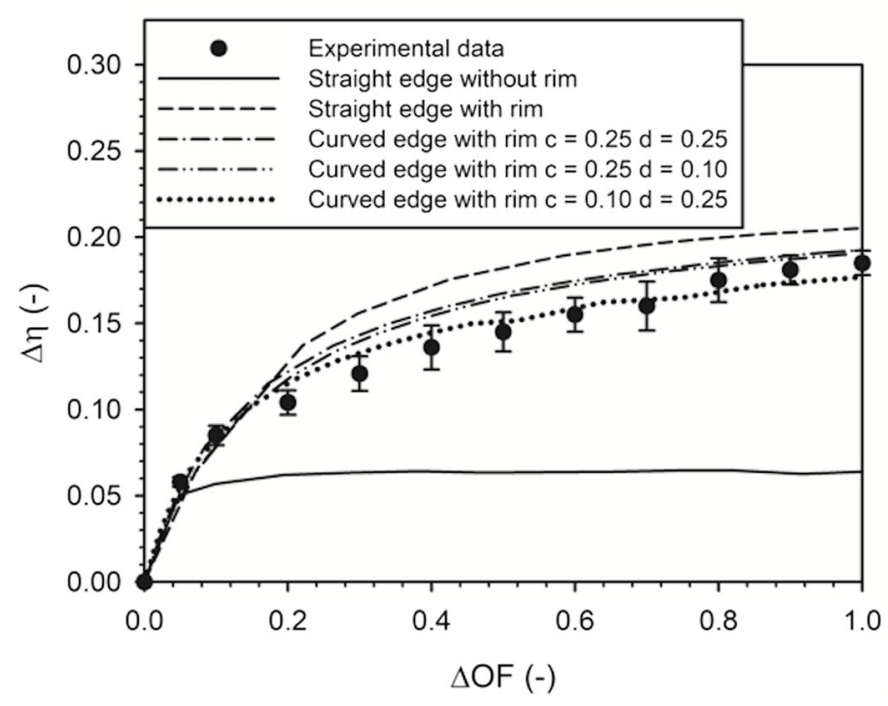

(b)
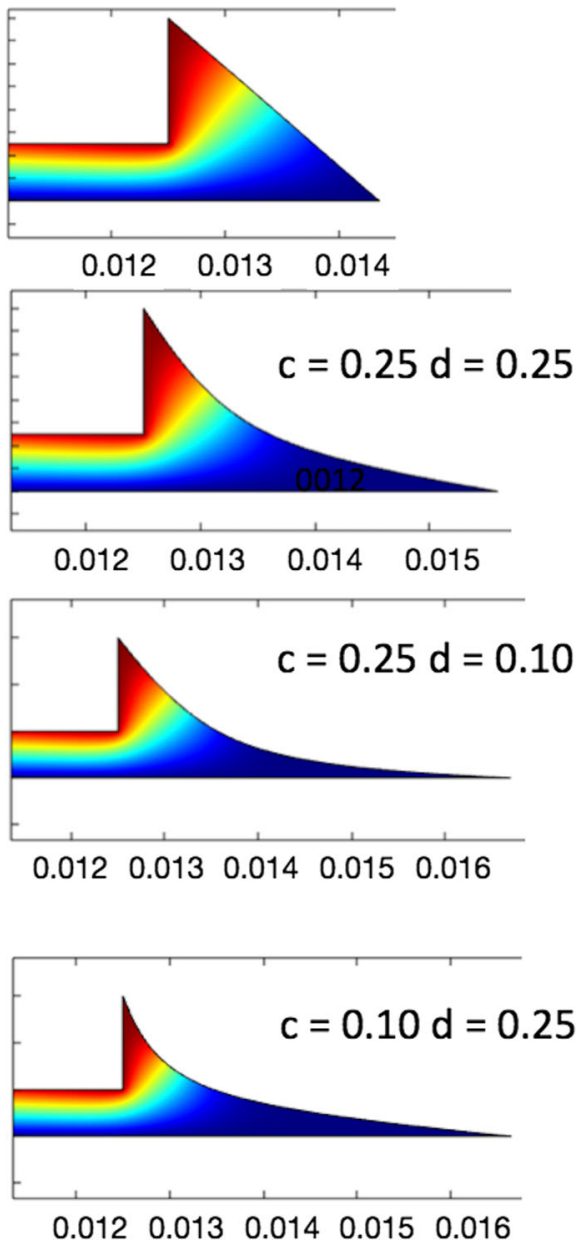

Fig. 6 a Comparison between experimental data and simulation results of viscosity error for $12.4 \mathrm{~Pa}$ s silicone oil in a PP setup with $D=$ $25 \mathrm{~mm}, H=0.5 \mathrm{~mm}$ and $H_{r}=1.1 \mathrm{~mm}$. The error bars indicate the

shape is taken into account, the numerical and experimental results correspond very well.

Since Fig. 6 shows that the applied approach provides a relatively good prediction of the measured viscosity error, the numerical simulations are subsequently used to obtain the flow field in the sample. First, it should be mentioned that for all the cases investigated here, the only non-zero component of the velocity field was that in the angular direction. Hence, no secondary flows were present. The origin of the increase of the measured torque values with overfilling can then be explored from plots of the shear rate throughout the gap. As long as Stokes flow is present in the gap, the flow profiles in the gap are similar, irrespective of the applied shear rate. Hence, only results for a shear rate of $1.0 \mathrm{~s}^{-1}$ will be shown here. Figure 7 shows the shear rate in the parallel plate setup for a gap of $1.0 \mathrm{~mm}$. The shear rates are multiplied with the square of the radius to allow assessing the relevant torque contributions as the area underneath the curves, according to Eq. 10. Figure 7a provides the rescaled shear rates as a function of the radial standard deviation on the data obtained from repeat measurements. b Edge shapes and velocity distributions at $\Delta \mathrm{OF}=0.5$

position $r$. For exact sample filling, the shear rate increases linearly, i.e., from $0.0 \mathrm{~s}^{-1}$ at the center to $1.0 \mathrm{~s}^{-1}$ at the edge, as expected for a parallel plate setup. When overfilling is present, the shear rate in the fluid at radial distances close to the plate radius increases rapidly to values larger than $1 \mathrm{~s}^{-1}$. This clearly shows that the presence of excess fluid due to overfilling affects the flow profile inside the gap. The increased shear rates at the measuring plate will increase the local shear stress and hence have a significant effect on the measured viscosity values, as can be seen in Figs. 4 and 5. In the range of studied amounts of overfilling, the distance over which the flow profile is affected does not depend on the amount of overfilling, but rather on the gap between the plates. This is illustrated in Fig. 7a, where the shear rate starts to deviate from the applied value of $1.0 \mathrm{~s}^{-1}$ at radial distances about $1 \mathrm{~mm}$ inwards from the plate edge, which roughly corresponds to the gap between the plates $(H=1.0 \mathrm{~mm})$. This observation confirms the generally accepted statement that edge effects in a finite geometry extend over a distance that corresponds to the gap height 


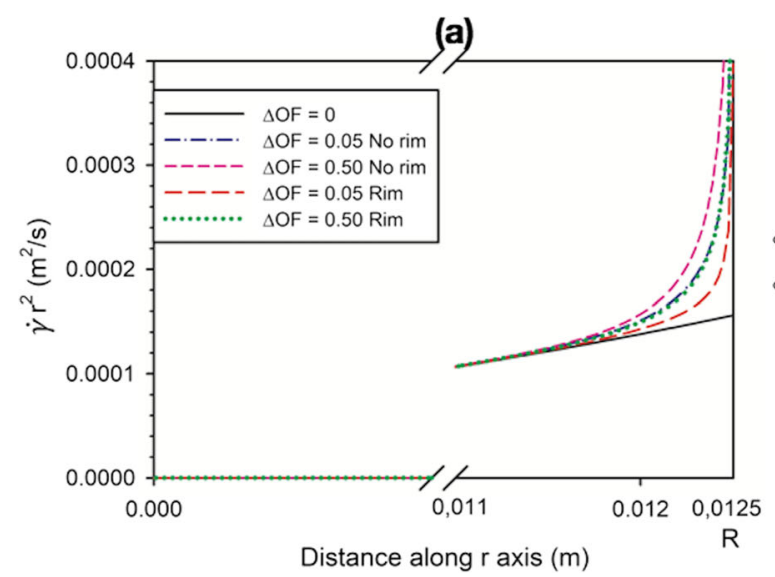

Fig. 7 Simulation results of the shear rate between the plates for an applied shear rate of $1.0 \mathrm{~s}^{-1}, D=25 \mathrm{~mm}, H=1 \mathrm{~mm}$ and $H_{r}=1 \mathrm{~mm}$, straight sample edge. a Shear rate at the bottom surface of the top plate.

(Tanner and Keentok 1983; Vrentas et al. 1991). Edge effects on the measured viscosity values can thus be minimized by using geometries with a large diameter to gap ratio. This is confirmed in Figs. 4 and 5, from which it clearly follows that the viscosity error is smaller for the plate with a larger diameter or when the gap height is smaller. A more detailed analysis of the effect of the diameter to gap ratio will be presented in the "Master curves for viscosity error" section.

Figure $7 \mathrm{a}$ also shows that, besides increasing with the amount of overfilling, the shear rate also increases more substantially when there is no rim wetting of the fluid. Hence, the corresponding torque contribution from the fluid underneath the upper plate is higher in absence of rim wetting. However, when the sample wets the rim, this also provides an extra torque contribution. This is shown in Fig. $7 \mathrm{~b}$, where the rescaled shear rates are provided as a function of the height at a radial distance that equals the plate radius. For an exact filling, the shear rate is equal to the applied shear rate of $1.0 \mathrm{~s}^{-1}$ across the complete gap height. If the simulations are run with overfilling, the shear rate across the gap at a radial distance that equals to the plate radius is not constant. It is lower than the applied value close to the bottom plate where the fluid extends further out of the gap and higher at the top plate where there is less excess fluid around the gap. The increase of the shear rate across the gap is slightly higher when the sample does not wet the plate rim. When the sample wets the rim, the stress along the plate rim is not zero and an extra torque contribution is generated by the presence of sheared sample along the rim. As can be seen from Fig. $7 b$, this torque contribution is significant and substantially increases in value and in the extent of the rim region over which it acts when the amount of overfilling is increased.

As mentioned before, the sample edge shape only depends on the surface forces and gravity and is independent

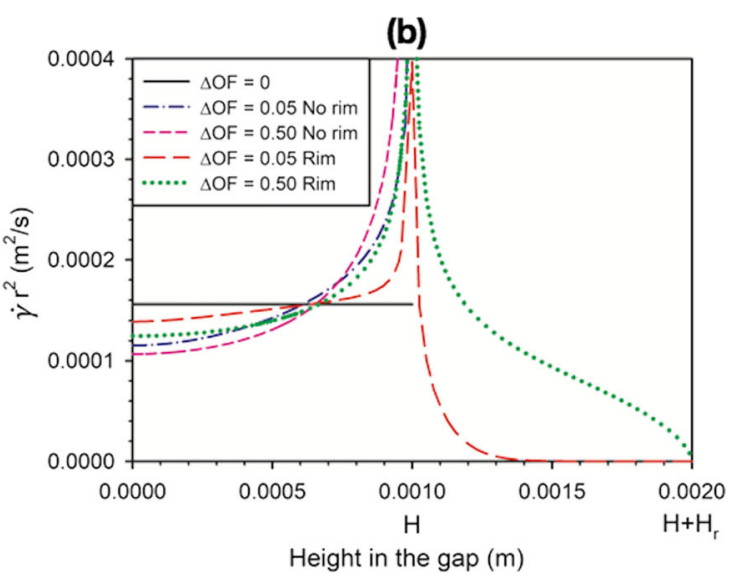

b Shear rate at a radial distance equal to the plate radius. Shear rates are rescaled to reflect the torque contributions (Eq. 10). The shear rate is taken as the magnitude of the rate of deformation tensor

of the viscous stresses in the material. Hence, the sample shape is expected to be independent of the sample viscosity. Moreover, in case of Stokes flow, the flow field in the sample also remains the same, irrespective of the sample viscosity. Hence, numerical simulations performed with different viscosity values, but with a fixed edge shape, revealed no effect of the sample viscosity on the viscosity error. Moreover, a selection of experiments performed with silicone oils of $0.99 \mathrm{~Pa} \mathrm{~s}$ and $101 \mathrm{~Pa} \mathrm{~s}$ also revealed no effect of the sample viscosity on the viscosity error. However, since the shape evolution towards steady state is much slower when the viscosity is higher, a longer waiting time should be implemented before the experimental measurement in case of high viscous liquids in order to obtain the steady state viscosity error.

\section{Master curves for viscosity error}

The viscosity error is caused by an extra torque $M_{e x}$ originating from the excess sample. To provide a uniform description of the overfilling error that is independent of the geometrical parameters, we derive a scaling relation. At first, we neglect the effect of rim wetting for our considerations. Thereto, we approximate the extra torque as proportional to an average additional stress $\sigma_{\text {out }}$ acting tangentially on the cylindrical area $2 \pi R H$ of the sample at a distance $R$ from the geometry center. This stress is assumed to be caused by the drag of the extra sample surrounding the cylindrical sample surface. It should be recognized that this stress and thus the extra torque is not so much determined by the overfilled volume but rather by the cross-sectional shape of the overfilled volume that surrounds the geometry. To capture the effect of overfilling shape rather than volume, the amount of overfilling, $\Delta \mathrm{OF}$, is rescaled to an effective $\Delta \mathrm{OF}_{\text {eff }}$ that reflects the shape and 
can thus be related to the relative extra torque $M_{\mathrm{ex}} / M$. For a certain overfilling volume, the overfilled shape depends on the geometry dimensions namely the gap height at the rim $H$ and the plate radius $R$. Keeping the same overfill shape, and hence the same $\Delta \mathrm{OF}_{\text {eff }}$, corresponds then to maintaining a constant ratio of radial to vertical dimension of the cross section, which can be written as $A_{\mathrm{ex}} / H^{2}$, with $A_{\mathrm{ex}}$ being the cross-sectional area of the overfilled volume. Since the cross-sectional area of the overfilled volume $A_{\mathrm{ex}} \simeq V_{\mathrm{ex}} /(2 \pi R)$, the ratio of the radial to vertical dimension of the overfilled volume can be expressed in terms of the excess volume, $A_{e x} / H^{2}=V_{e x} /\left(2 \pi R H^{2}\right)$. Inserting the expression from the "Definition of viscosity error and amount of overfilling" section for $V_{\text {geometry }}$ for a $\mathrm{PP}$ or CP setup results in:

$\Delta \mathrm{OF}_{\text {eff }}=\frac{R}{b H} \frac{V_{\text {ex }}}{V_{\text {geometry }}}=\frac{R}{b H} \Delta \mathrm{OF}$

in which $b=2$ for a PP setup and $b=3$ for a CP setup.

Further, the viscosity error can be calculated from the ratio of the extra torque $M_{\mathrm{ex}}$ due to the overfilling to the sample torque at correct sample filling $M$ of Eq. 11:

$\Delta \eta \sim \frac{M_{\mathrm{ex}}}{M}=\frac{2 \pi R^{2} H}{a^{-1} \pi R^{3}} \frac{\sigma_{\mathrm{out}}}{\sigma}=\frac{2 a H}{R} \frac{\sigma_{\mathrm{out}}}{\sigma}$

in which $\sigma$ is the sample shear stress for correct sample filling and $\sigma_{\text {out }}$ is the additional average shear stress resulting from the drag of the overfilled sample volume that should be a function of the overfilled shape and therefore a function of $\Delta \mathrm{OF}$. Both stresses $\sigma$ and $\sigma_{\text {out }}$ scale linearly with the sample viscosity $\eta_{0 \%}$ and the shear rate at radius $\mathrm{R}, \dot{\gamma}=\Omega R / H$. However, $\sigma_{\text {out }}$ will also exhibit a (nonlinear) dependency on the shape of the overfilled volume. Simple geometrical arguments show that for the same shape (expressed by a constant ratio $A_{e x} / H^{2}$ as shown for the derivation of Eq. 12) a single relative stress $\sigma_{\text {out }} / \sigma$ will be observed, independent of the absolute magnitude of $A_{\text {ex }}$ or $H$. This means that we can describe the shape dependency of the relative stress $\sigma_{\text {out }} / \sigma$ with a single function $f_{\text {shape }}$ that depends solely on the effective overfilling of Eq. 12. Hence, the viscosity error can be expressed as:

$\Delta \eta=\frac{2 a H}{R} f_{\text {shape }}\left(\frac{R}{b H} \Delta \mathrm{OF}\right)$

Based on this derivation, plotting a rescaled viscosity error $\frac{\Delta \eta R}{2 a H}$ as a function of the effective overfilling $\frac{R}{b H} \Delta \mathrm{OF}$ should collapse all curves of viscosity error onto a single master curve. First, this scaling is performed for numerical simulation results in which PP and CP setups are used with various diameters, gap heights and cone angles. The numerical results, using the straight edge configuration of Fig. 2b, are shown in Fig. 8a. Similar to the experimental data, the overfilling error is shown to increase with increasing gap height at the edge and with decreasing plate diameter. After rescaling with the scaling factors proposed in Eq. 14, Fig. 8b demonstrates that the curves of the different overfilling scenario's in PP and CP setups without rim wetting collapse relatively well. The master curve in Fig. $8 \mathrm{~b}$ follows an exponential rise profile given by:

$f_{\text {shape }}=0.4\left(1-\exp \left(-2.5 \frac{R}{b H} \Delta \mathrm{OF}\right)\right)$

This also allows to approximate a maximum viscosity error that can be expected for a certain geometry. From the observed plateau of $\frac{\Delta \eta R}{2 a H}$ at higher overfilling one can read off the maximum $f_{\text {shape }}$ that correlates the maximum viscosity error $\Delta \eta_{\max }$ and the geometry parameters $2 a H / R$ so that:

$\Delta \eta_{\max }=f_{\text {shape, } \max } \frac{2 a H}{R}$

From Fig. 8 and Eq. 15 , it is clear that $f_{\text {shape,max }} \approx 0.4$ so that $\Delta \eta_{\max }=0.8 a H / R$ for cases without rim wetting. This scaling relation clearly demonstrates the previously mentioned increase of the viscosity error with gap height and decrease with geometry radius. Moreover, it also clearly shows that when the radius is the same, the viscosity error in a plate-plate setup will in general be higher than that in a cone-plate setup due to two effects. First, the prefactor $a$ is higher for a PP than for a CP setup, which reflects that
Fig. 8 a Simulation results of the viscosity error versus the amount of overfilling in various plate-plate and cone-plate setups without rim wetting and with a straight sample edge. b Rescaled simulation results (a)
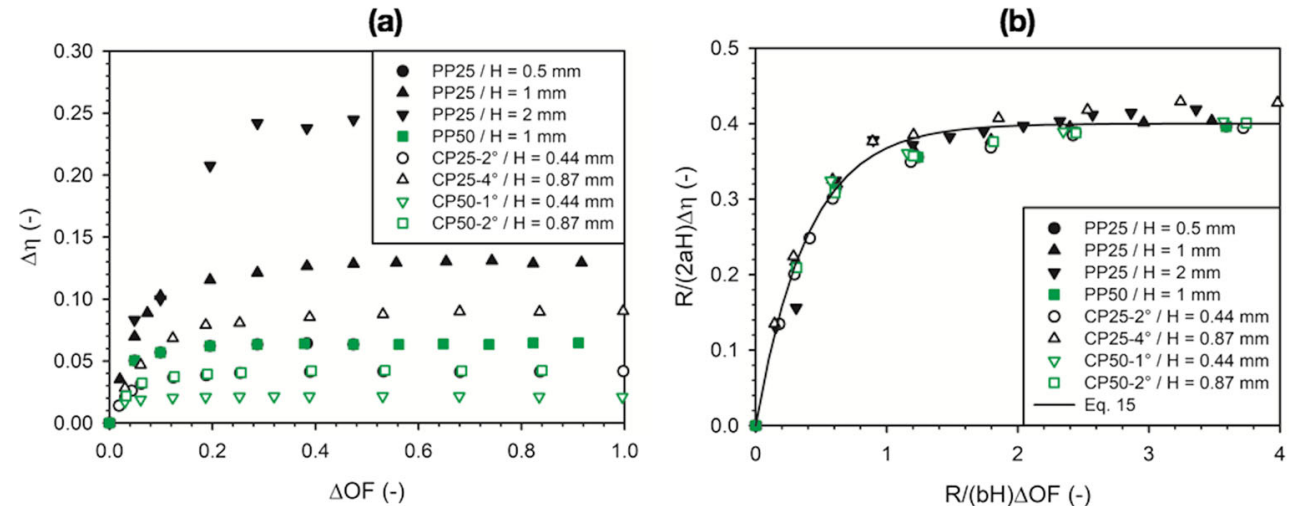
for the same geometry radius $R$ a PP geometry creates less sample torque than a $\mathrm{CP}$ geometry at the same height $H$, so that the additional torque from overfilling weighs stronger. Second, the gap height $H$ at the edge is in general also larger for a PP setup than for a CP setup. It can be concluded that the presented scaling approach captures the effects of the (known) dimensions of the geometry and allows to compare the effects of effective overfillings (and the shape of the extra volume) for geometries of different types, radii, and gap heights. It should be noted that, as can be expected from Fig. 6, edge shapes with a higher curvature result in a slightly lower value of $f_{\text {shape, max }}$. However, superposition of the data for different geometries remains valid as long as the edge shape, as presented in Eqs. 6 and 7, is kept constant (data not shown).

During the experiments, it was observed that, when loading a sample to an overfilled condition, the sample was forced to break the pinning to the lower edge of the upper geometry and to wet the geometry's outer rim. This rim wetting upon overfilling is generally taking place when applying the trimming procedure, during which already the scraping of the edge leads to a partial wetting of the rim. During the approach of the final measuring gap, the sample is then fully wetting the rim and pinning to the upper edge, as shown in Fig. 3. In the case of a wetting of the outer rim and pinning to the upper edge, Eq. 14 needs to be adjusted accordingly. The vertical dimension of the cross-sectional area $A_{\text {ex }}$ of the overfilled volume $V_{\text {ex }}$ is in the case of rim wetting the sum of the sample height $H$ and the height or "thickness" of the rim $H_{\mathrm{r}}$. Hence, the definition of the effective overfilling that enters the shape function $f_{\text {shape }}$ should be adapted. Since the conservation of the overfilled shape requires now $A_{\text {ex }} /\left(H+H_{r}\right)^{2}$ to be constant, Eq. 14 will contain a correction factor $H /\left(H+H_{\mathrm{r}}\right)^{2}$, so that it takes on the form:

$\Delta \eta=\frac{2 a H}{R} f_{\text {shape }}\left(\frac{R H}{b\left(H+H_{\mathrm{r}}\right)^{2}} \Delta \mathrm{OF}\right)$

Moreover, the tangential stress exerting a drag force on the sample due to the overfilled sample now acts over a height
$H+H_{r}$ rather than $H$. However, also the average additional stress $\tau_{\text {out }}$ scales now with a shear rate that depends on $H+H_{r}$, so that the height in the prefactor of the right-hand side of Eq. 14 does not change. Equation 17 thus presents a scaling relation that should take into account rim wetting. This scaling was applied to results of numerical simulations for plate-plate setups with various gap and rim heights, as shown in Fig. 9. It can be seen that this scaling indeed provides a rather good collapse of data obtained at various total heights $H+H_{r}$. However, a pronounced effect of the ratio of gap height to total height $x=H /\left(H+H_{\mathrm{r}}\right)$ remains. This can be rationalized by the fact that the velocity field in the overfilled volume will depend on how much fluid is in contact with the rim height $H_{\mathrm{r}}$, and how much with the sample over the height $H$. Without rescaling of the viscosity error according to Eq. 17, the viscosity error shows a nonmonotonous dependence on the ratio $x=H /\left(H+H_{\mathrm{r}}\right)$. However, after rescaling with $H$, as shown in Fig. 9a, the viscosity error shows a monotonous dependence on $x$. As a matter of fact, it turns out that weighing the shape function $f_{\text {shape }}$ with a correction factor $x+(1-x) k$ that incorporates the ratio $x=H /\left(H+H_{r}\right)$ and a weighing coefficient $k$ allows to take into account the relative contributions of both parts of the overfilled volume. Hence, Eq. 17 is corrected to read as:

$$
\Delta \eta=\frac{2 a H}{R}[x+(1-x) k] f_{\text {shape }}\left(\frac{R H}{b\left(H+H_{\mathrm{r}}\right)^{2}} \Delta \mathrm{OF}\right)
$$

Determining the unknown $k$ as a fit parameter from a set of simulation data with $H+H_{\mathrm{r}}=1 \mathrm{~mm}$ leads to $k=4.3$. Subsequently, simulation data with a straight edge shape and various gap heights can be overlapped rather well on a master curve by plotting $\frac{\Delta \eta R}{2 a H[x+(1-x) k]}$ as a function of $\frac{R H}{b\left(H+H_{\mathrm{r}}\right)^{2}} \Delta \mathrm{OF}$ in Fig. 9b. It should be noted that this scaling only holds for $x \geq 0.3$ whereas for lower values of $x$, the linear scaling of the overfilling error with $x$ breaks down. However, for typical experimental geometrical parameters
Fig. 9 Simulation results of the viscosity error versus the amount of overfilling in a plate-plate setup with $D=25$ $\mathrm{mm}$ with rim wetting and with a straight sample edge. a Rescaled according to Eq. 17. b Rescaled according to Eq. 18
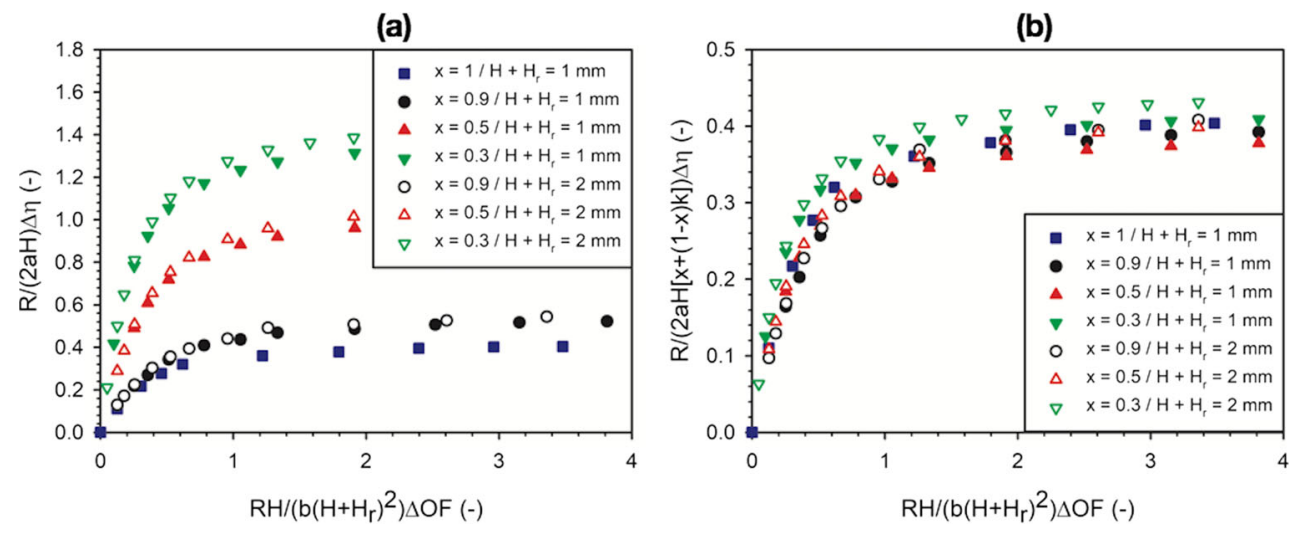


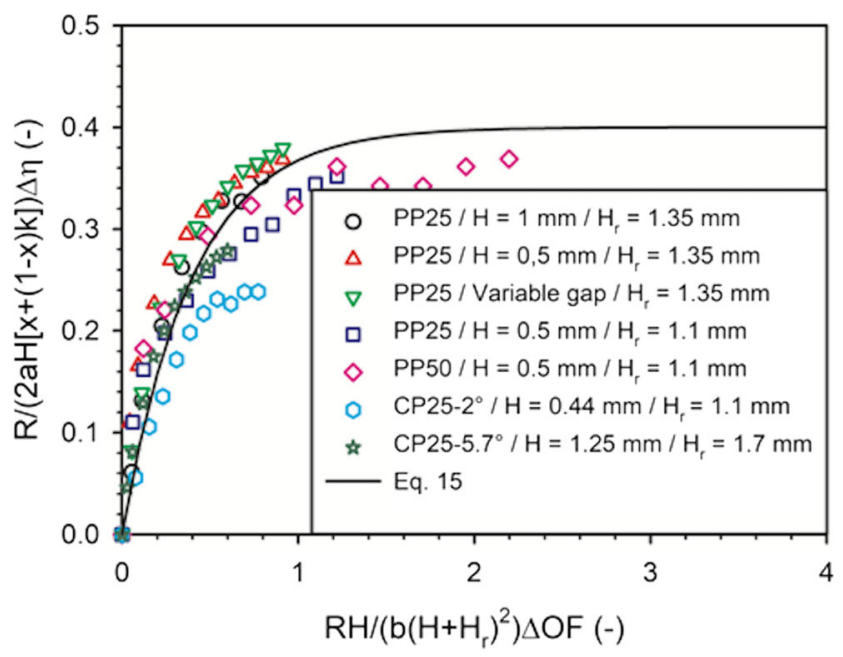

Fig. 10 Experimental results of the viscosity error versus the amount of overfilling in various plate-plate and cone-plate setups after rescaling according to Eq. 18

$H$ and $H_{r}$ as given in Figs. 4 and 5, the requirement of $x \geq 0.3$ is maintained and the master curve of Fig. 9 holds.

Based on the scaling arguments presented here, a rescaling of the experimental data in Figs. 4 and 5 can be performed. The result is shown in Fig. 10. From this figure, it can be concluded that the proposed scaling approach allows to capture the main trends of the effects of the geometrical parameters on the overfilling error in rotational rheometry. However, since the model is based on a representation of the overfill shape by its ratio of vertical to horizontal dimension, which does not uniquely capture shape effects due to the possibility of different curvatures of the edge shape as a function of the amount of overfilling, the description is not exact. To capture these secondary effects, it is expected that more details of the sample shape should be taken into account, which could possibly be done by applying an additional scaling with the Bond number (Bo $=\rho g L_{c}^{2} / \alpha$, with $\rho$ the fluid density, $g$ the gravitational constant, $L_{c}$ the characteristic length scale and $\alpha$ the fluid surface tension). When taking the sum of the gap height $H$ and rim height $H_{r}$ as the relevant length scale in the Bond number, it varies between 1.1 and 4.1 for the different cases presented in Fig. 10. Hence, differences in sample edge shape are expected between the different samples due to different amounts of sagging under the effect of gravity. This more detailed analysis of the edge shape is, however, beyond the scope of the present work, in which we focus on the primary effects of the geometrical parameters on the viscosity error due to overfilling. It should be noted that from Fig. 6 it becomes clear that differences in the sample edge shape will only provide minor corrections to the proposed scaling model, at least for Newtonian samples.

\section{Conclusions}

The error on the measured viscosity due to overfilling of Newtonian liquids in rotational rheometry has systematically been investigated. The error in viscosity increases steeply for small amounts of overfilling before reaching a plateau value at high amounts of overfilling. Overfilling simulations revealed that there is a large torque contribution from the overfilled sample that is pinned to the rim of the upper geometry. The plateau value of the measurement error is determined by the ratio between the gap height and the plate or cone radius. Moreover, with the same gap height at the edge, the viscosity error in a cone-plate setup is only $75 \%$ of that obtained with a parallel plate setup. By rescaling the amount of overfilling to a fixed shape of the overfilled volume and deriving the extra torque due to overfilling from scaling arguments, a universal relation for the evolution of the viscosity error with the amount of overfilling was derived. This relation captures the main effects of the geometrical parameters on the viscosity error and allows to predict viscosity errors a priori for a certain geometrical setup. Thereby, our work will allow to correct for viscosity errors due to overfilling, allowing to introduce overfilling as a solution for problems such as edge fracture or solvent evaporation without loss of measurement accuracy.

\section{Recommendations}

In general, several recommendations can be made to avoid or minimize the errors due to sample overfilling:

1. For parallel plate setups, a measuring geometry with a large diameter should be used at a small measuring gap. For cone-plate setups, a small measuring gap at the geometry edge is realized by using a small cone angle. At small gaps, parallelism will become important and therefore care must be taken to align the geometry properly. Moreover, during sample loading in parallel plate geometries, air bubbles might be formed (Reddy 2019). To avoid formation of air bubbles, the center of the upper plate should be wetted with a small amount of sample before sample loading so as to induce a forced wetting from the center.

2. During sample loading, wetting of the outer rim of the measuring plate should be avoided. This alone will reduce the measurement error to a large extent. If this cannot be avoided, then measuring geometries with a thin edge should be used.

3. In cone-plate setups, the sample should be scraped at a correct trimming height, as defined in Eq. 5, to result in a spherical sample edge shape, when lowered to the correct measurement height. With low viscous and 
low surface tension fluids, care should be taken not to remove too much sample during scraping as this will cause underfilling. Alternatively, the required sample volume can be calculated and directly added with an accurate volumetric pipette, thereby avoiding the need for sample trimming.

4. When using a bottom plate with dimensions equal to that of the top plate, lower amounts of overfilling can be reached, as the sample will flow out at high amounts of overfilling. Therefore, the effects of overfilling can be reduced with such a setup. It is expected that due to the different shapes of the sample surface, the overall scaling presented in Eq. 18 will remain valid, but the exact value of the viscosity error, determined by the fitting parameter $k$, will be different.

5. The analysis performed here is applicable for sample overfilling. However, it is known that sample underfilling leads to a viscosity value that is lower than the true value (Davies 2015; Hellström et al. 2015). In the case of symmetric underfilling, the correct viscosity value can be obtained by taking into account that the generated torque only originates from the part of the geometry that is wetted by the sample. By taking the wetted radius $R_{\mathrm{W}}$ as the radius of the sample edge and defining the amount of underfilling $\Delta \mathrm{UF}$ as in Eq. 2, the viscosity error due to underfilling in a plate-plate geometry as derived by Hellström et al. (2015) can be rewritten as:

$\Delta \eta=\left(\frac{R_{\mathrm{w}}}{R}\right)^{4}-1=\Delta \mathrm{UF}\left(1+\left(\frac{R_{w}}{R}\right)^{2}\right)$

This relation was experimentally verified by Hellström et al. (2015) for small amounts of underfilling. From this analysis, it can be seen that, contrary to overfilling effects, underfilling effects are not dependent on the ratio of the gap height to geometry radius. Hence, for certain geometries, underfilling effects can be more severe than overfilling effects. However, since the viscosity errors due to underfilling are not expected to level off at a certain amount of underfilling, they will always become larger than errors due to overfilling when deviations from the correct amount of filling are substantial.

6. In principle, any extra fluid in contact with the geometry or the sample will result in an additional torque contribution. For instance, using a low viscous fluid around the sample to avoid evaporation will also cause overfilling effects, be it much less severe than overfilling with the actual sample due to the lower viscosity and thus lower extra torque contribution. Moreover, the use of an evaporation blocker with a vapor lock connected to the geometry will increase the measured torque. However, when keeping in mind that the solvent in the solvent reservoir in general has a much lower viscosity as compared with the sample and that both the immersed surface area and the radial distance from the centerline are much smaller for the vapor lock as compared with the measuring geometry, this contribution can most often be neglected. If not, this torque contribution can be measured a priori and can subsequently be subtracted from the measured torque of the sample.

\section{Outlook}

In the present work, the effects of overfilling on viscosity measurements of Newtonian fluids are studied. In that case, the relative error in viscosity is independent of the sample viscosity and thus plays an equal role for low and high viscous materials. However, for fluids that exhibit shear thinning/thickening or viscoelasticity, additional effects may come into play. Qualitatively, it is expected that the increase of the shear rate at the geometry edge due to overfilling will reduce the viscosity error due to the overfilling for shear thinning fluids. However, preliminary experiments on viscoelastic fluids also revealed that viscoelasticity affects the sample edge shape, which also contributes to the overfilling effects. Moreover, Shipman et al. (1991) have shown that the surface shape contributes more strongly to normal force measurements as compared with shear stress measurements in rotational rheometry. Unravelling these additional effects is beyond the scope of the present work, but it is clear that, in addition to the effects for Newtonian fluids, the viscoelastic sample properties will further affect the measured viscosity error due to overfilling.

Traditionally, rheological measurements required the generation of rheometric flows, i.e., pure shear or extensional flows with a homogeneous shear or extension rate throughout the sample. However, ongoing developments concerning localized characterizations of deformation fields as well as combined experimental-numerical approaches expand the possibilities for performing material characterization in non-model flow conditions. This will allow in situ material characterization or characterization of small quantities of materials that do not allow loading of standard geometry setups. Our work is a simple example of how scaling and combination of experiments with numerical simulations can allow to extract accurate measurement data even in absence of a well-defined sample geometry.

Acknowledgments The undergraduate student R. Blok (TU Eindhoven) is thanked for his contributions to the experiments. The authors thank Prof. J. Mewis for fruitful discussions and would like to dedicate this paper to him for his 81 st birthday. 
Funding information This study received financial support from the SoPPom program of the Flemish Strategic Initiative for Materials (SIM) and the Research Foundation Flanders (FWO) under grant G077916N.

Open Access This article is distributed under the terms of the Creative Commons Attribution 4.0 International License (http:// creativecommons.org/licenses/by/4.0/), which permits unrestricted use, distribution, and reproduction in any medium, provided you give appropriate credit to the original author(s) and the source, provide a link to the Creative Commons license, and indicate if changes were made.

\section{References}

Choi C-H, Kim C-J (2006) Large slip of aqueous liquid flow over a nanoengineered superhydrophobic surface. Phys Rev Lett 96:066001-5

Clasen C, McKinley GH (2004) Gap-dependent microrheometry of complex liquids. J Non-Newt Fluid Mech 124:1-10

Clasen C, Gearing BP, McKinley GH (2006) The flexure-based microgap rheometer (FMR). J Rheol 50:883-905

Davies AJ (2015) Loading errors in cone-plate rheometry. Meas Sci Technol 26:127001/1-3

Giles DW, Hooper RW (1999) Excess Edge effect in rotational parallel plate rheometry. Presentation at Society of Rheology 71st Annual meeting, 17-21 October 1999, Madison (Wisconson)

Griffiths DF, Jones DT, Walters K (1969) A flow reversal due to edge effects. J Fluid Mech 36:161-175

Griffiths DF, Walters K (1970) On edge effects in rheometry. J Fluid Mech 42:379-399

Hellström LHO, Samaha MA, Wang KM, Smits AJ, Hultmark M (2015) Errors in parallel-plate and cone-plate rheometer measurements due to sample underfill. Meas Sci Technol 26:015301/1-4

Joh SW, Lee SH, Youn JR (2010) Rheological behaviour of polydispersed bubble suspensions in shear flows. Polym Eng Sci 50:128-137

Johnston MT, Ewoldt RH (2013) Precision rheometry: surface tension effects on low-torque measurements in rotational rheometers. J Rheol 57:177-195

Kalika DS, Nuel L, Denn MM (1986) Gap-Dependence Of the viscosity of a thermotropic liquid crystalline copolymer. J Rheol 33:1059-1070
Khaliullin RN, Schieber JD (2009) Self-Consistent Modeling of constraint release in a Single-Chain Mean-Field Slip-Link model. Macromolecules 42:7504-7517

Larson RG (1999) The Structure and Rheology of Complex Fluids. Oxford University Press, New York

Macosko CW (1994) Rheology Principles Measurements and Applications. VCH, New York

Paddon DJ, Walters K (1979) On edge effects and related sources of error in rotational rheometry. Rheol Acta 18:565-575

Pieper S, Schmid HJ (2016) Guard ring induced distortion of the steady velocity profile in a parallel plate rheometer. Appl Rheol 26:64533/1-7

Quinzani LM, Vallés EM (1986) The use of a modified cone-and-plate geometry (MCP) in a rotational rheometer for the measurement of material functions. J Rheol 30(s):s1-s21

Reddy N (2019) Bubble formation during sample loading in rotational rheometers, Vimeo, https://vimeo.com/337228429

Schweizer T, Schmidheiny W (2013) A cone-partitioned plate rheometer cell with three partitions (CPP3) to determine shear stress and both normal stress differences for small quantities of polymeric fluids. J Rheol 57:841-856

Sharma V, Jaishankar A, Wang YC, McKinley GH (2011) Rheology of globular proteins: apparent yield stress, high shear rate viscosity and interfacial viscoelasticity of bovine serum albumin solutions. Soft Matter 7:5150-5160

Shipman RWG, Denn MM, Keunings R (1991) Free-surface effects in torsional parallel-plate rheometry. Ind Eng Chem Res 30(5):918922

Snijkers F, Vlassopoulos D (2011) Cone-partitioned-plate geometry for the ARES rheometer with temperature control. J Rheol 55:1167-1186

Srinivasan S, Choi W, Park K-C, Chhatre SS, Cohen RE, McKinley GH (2013) Drag reduction for viscous laminar flow on SprayCoated Non-Wetting surfaces. Soft Matter 9:5691-5702

Tanner RI, Keentok M (1983) Shear fracture in cone and plate rheometry. J Rheol 27:47-57

Tran-Duc T, Phan-Thien N, Khoo C (2013) Rheology of bubble suspensions using dissipative particle dynamics. Part i: A hardcore DPD particle model for gas bubbles. J Rheol 57:1715-1737

Vrentas JS, Venerus DC, Vrentas CM (1991) An exact analysis of reservoir effects for rotational viscometers. Chem Eng Sci 46:3337

Walters K (1975) Rheometry. Chapman and Hall, London

Wu S (1982) Polymer interface and adhesion. Marcel Dekker Inc., New York

Publisher's note Springer Nature remains neutral with regard to jurisdictional claims in published maps and institutional affiliations. 